\title{
Estudio retrospectivo comparativo entre aspirina y dabigatrán en la incidencia de eventos tromboembólicos en pacientes con artroplastia de cadera y rodilla
}

\author{
Fernando A. Lopreite, Tamara Dainotto, Stéfano Gaggiotti, Hernán del Sel \\ Equipo de Cadera y Rodilla, Hospital Británico de Buenos Aires, Ciudad Autónoma de Buenos Aires, Argentina
}

\begin{abstract}
RESUMEN
Objetivo: Analizar la incidencia de eventos tromboembólicos extrahospitalarios luego de un reemplazo total de rodilla o cadera primario en los primeros 90 días posoperatorios, utilizando como tromboprofilaxis ácido acetilsalicílico o dabigatrán. El objetivo secundario fue evaluar el costo de la terapia antiagregante y de la anticoagulante. Materiales y Métodos: Se realizó un estudio observacional retrospectivo en el posoperatorio de reemplazos totales de cadera y rodilla primarios sobre la incidencia de eventos tromboembólicos extrahospitalarios, durante los primeros 90 días posteriores a la cirugía, en dos grupos. Se administró ácido acetilsalicílico (325 mg/día) o dabigatrán (150-220 mg/día) por 35 días. Resultados: La serie incluyó a 224 pacientes (media de la edad 68.5 años), el $44,2 \%$ eran hombres. El $51,3 \%$ era un reemplazo total de cadera. El $64,3 \%$ continuó la tromboprofilaxis con dabigatrán y el $35,7 \%$, con aspirina. La incidencia total de eventos tromboembólicos fue del 1,3\%; 1,4\% con dabigatrán y 1,3\% con aspirina $(p=0,9)$. El costo de la tromboprofilaxis fue de USD 3,6 con aspirina $325 \mathrm{mg}$ y USD 130 o 175 con dabigatrán, según la presentación de 75 y $110 \mathrm{mg}$. Conclusiones: La aspirina como tromboprofilaxis tras un reemplazo total de cadera o rodilla en pacientes con bajo riesgo de sufrir eventos tromboembólicos ha logrado resultados clínicos similares a los del dabigatrán. Se puede recomendar un protocolo multimodal basado en el uso de aspirina. Este mejora el cumplimiento de la terapia antitrombótica por parte de los pacientes, debido al bajo costo de la profilaxis con aspirina.
\end{abstract}

Palabras clave: Aspirina; ácido acetilsalicílico; dabigatrán; tromboprofilaxis; evento tromboembólico; artroplastia de cadera; artroplastia de rodilla.

Nivel de Evidencia: III

\section{Comparative Retrospective Study Between Aspirin and Dabigatran in the Incidence of Thromboembolic Events in Hip and Knee Arthroplasty}

\begin{abstract}
Objective: To analyze the incidence of out-of-hospital thromboembolic events after a primary total knee or hip replacement in the first 90 postoperative days, using acetylsalicylic acid or dabigatran as thromboprophylaxis. As a secondary objective, to evaluate the cost of antiplatelet therapy compared to anticoagulants. Materials and Methods: A retrospective observational study was carried out in the postoperative period of primary total hip and knee replacement on the incidence of out-of-hospital thromboembolic events during the first 90 postoperative days in two groups of patients. Acetylsalicylic acid (325 mg per day) or dabigatran (150 to $220 \mathrm{mg}$ per day) were used as thromboprophylaxis for 35 days. Results: The series consisted of 224 patients aged 68.5 years (38-95 years), $44.2 \%$ male. $51.3 \%$ corresponded to total hip replacement. $64.3 \%$ continued thromboprophylaxis with dabigatran and $35.7 \%$ with aspirin. The total incidence of thromboembolic events was $1.3 \%$. In the patients who received dabigatran it was $1.4 \%$ and in those who received aspirin, $1.3 \%(p=0.9)$. The cost of thromboprophylaxis with aspirin $325 \mathrm{mg}$ was US \$ 3.6 while with dabigatran it was US $\$ 130$ or $\$ 175$, according to its presentation, 75 and $110 \mathrm{mg}$. Conclusion: Aspirin as thromboprophylaxis after total hip or knee replacement in individuals at low risk of thromboembolic events has shown similar clinical outcomes as dabigatran, and a multimodal protocol based on the use of aspirin can be recommended. This improves patient adherence to antithrombotic therapy, due to the low cost of aspirin prophylaxis.
\end{abstract}

Keywords: Aspirin; acetylsalicylic acid; dabigatran; thromboprophylaxis; thromboembolic event; hip arthroplasty; knee arthroplasty. Level of Evidence: III

Recibido el 20-1-2021. Aceptado luego de la evaluación el 8-3-2021 • Dr. FERNANDO A. LOPREITE • fernandolopreite @ hotmail.com

https://orcid.org/0000-0002-2065-8649

Cómo citar este artículo: Lopreite FA, Dainotto T, Gaggiotti S, del Sel H. Estudio retrospectivo comparativo entre aspirina y dabigatrán en la incidencia de eventos tromboembólicos en pacientes con artroplastia de cadera y rodilla. Rev Asoc Argent Ortop Traumatol 2021;86(4):446-454. https://doi.org/10.15417/issn. 1852-7434.2021.86.4.1303 


\section{INTRODUCCIÓN}

A pesar de que las artroplastias totales de cadera y rodilla son procedimientos frecuentes y seguros, se considera que los pacientes que se someten a ellas tienen un alto riesgo de sufrir complicaciones tromboembólicas, como tromboembolismo pulmonar (TEP) y trombosis venosa profunda (TVP). ${ }^{1,2}$ Según la bibliografía, la incidencia posoperatoria de estas complicaciones oscila entre el $15 \%$ y el 30\% para la TVP, y entre el 1,7\% y el 7,8\% para el TEP, sin profilaxis. ${ }^{1-3}$ La tromboprofilaxis con fármacos anticoagulantes después de estos procedimientos ha disminuido un 0,6-2\% la tasa de eventos tromboembólicos (ETE), según diferentes reportes. ${ }^{3-5}$

Para tal fin, existen múltiples tratamientos farmacológicos, entre ellos, los inhibidores del factor Xa (rivaroxabán, apixabán), los inhibidores de la trombina, como el dabigatrán, la heparina de bajo peso molecular y los antagonistas de la vitamina $\mathrm{K}$ (warfarina). ${ }^{6}$ Asimismo, en los últimos años, se ha observado un auge de la administración de fármacos antiagregantes, como el ácido acetilsalicílico (AAS), ante la publicación de ensayos clínicos y metanálisis que sugieren que este agente posee similares tasas de efectividad que los anticoagulantes para la prevención de la TVP y el TEP. ${ }^{1,3-5,7,8}$

En 2009, el AAS fue incorporado como recomendación en la profilaxis antitrombótica para el posoperatorio de un reemplazo total de cadera (RTC) o de rodilla (RTR) por la guía de la American Academy of Orthopaedic Surgeons (AAOS). ${ }^{9}$ Posteriormente, en 2012, la guía del American College of Chest Physicians (ACCP) enumera este fármaco junto con otros agentes tromboprofilácticos (heparina de bajo peso molecular, dabigatrán, rivaroxabán, entre otros) con recomendación grado 1B para su uso como profilaxis de ETE. ${ }^{10}$ Desde entonces, el porcentaje de cirujanos que decide emplearla como única droga para la tromboprofilaxis aumentó de forma significativa, con similares tasas de ETE que con la administración de fármacos anticoagulantes. ${ }^{4}$

Por otro lado, el AAS se presenta como un fármaco con un alto perfil de seguridad, fácil de administrar, bien tolerado y accesible tanto por sus costos como por su disponibilidad, con poder analgésico, antinflamatorio y antiagregante plaquetario, que juega un rol central en la prevención de enfermedades cardiovasculares. ${ }^{1,2,11,12}$ Esto asociado a los resultados favorables como profilaxis antitrombótica lo convierten en una alternativa segura y atractiva para la comunidad médica. Asimismo, numerosos estudios han demostrado el efecto cardioprotector del AAS, lo cual agregaría un beneficio a su uso como antitrombótico. ${ }^{12}$

En nuestro Centro, se prescriben habitualmente agentes anticoagulantes orales, como dabigatrán, o subcutáneos (enoxaparina) al alta hospitalaria, por un mínimo de 35 días. ${ }^{13}$ Recientemente comenzamos a usar el AAS como monodroga en la profilaxis extrahospitalaria de ETE durante el posoperatorio de cirugías de reemplazo articular, tras haber observado un menor cumplimiento del tratamiento farmacológico con los agentes anticoagulantes habituales debido a los mayores costos que representa para los pacientes y las potenciales consecuencias que eso conlleva.

El principal objetivo de este estudio fue analizar, en forma retrospectiva, la incidencia de TVP y TEP extrahospitalarios en dos grupos de pacientes: grupo I (AAS) y grupo II (dabigatrán) luego de un RTR o RTC primario, en los primeros 90 días después de la cirugía. Como objetivos secundarios, se buscó determinar las tasas de complicaciones hemorrágicas y de reintervención asociadas al uso de dichos fármacos y analizar el costo de la terapia antiagregante (grupo I) y de la anticoagulante (grupo II).

Nuestra hipótesis es que la incidencia de ETE en nuestro Centro es similar con AAS o con dabigatrán en pacientes con un bajo riesgo de sufrir un ETE, teniendo en cuenta que el costo económico de la profilaxis con AAS es inferior al de dabigatrán, lo que mejoraría el cumplimiento por parte de los pacientes.

\section{MATERIALES Y MÉTODOS}

Entre el 1 de mayo de 2019 y el 20 de abril de 2020, se llevó a cabo un estudio retrospectivo para evaluar la incidencia de ETE extrahospitalarios durante los primeros 90 días posteriores a un RTC o RTR primario unilateral, programado que incluyó a los pacientes operados, de manera consecutiva, a quienes se dividió en dos grupos. A los del grupo I se les indicó como tromboprofilaxis AAS $325 \mathrm{mg}$ /día y a los del grupo II, dabigatrán 150-220 mg/día en función de la edad y la depuración de creatinina, hasta completar 35 días de profilaxis tanto para el RTC como para el RTR.

Uno de los cirujanos del equipo decidió indicar AAS a todos sus pacientes que cumplieran los criterios de inclusión y el resto de los cirujanos continuaron con dabigatrán como medicación profiláctica para todos sus pacientes.

Los criterios de inclusión fueron: edad $>18$ años, artroplastia primaria de cadera o de rodilla como tratamiento de la artrosis, profilaxis extrahospitalaria con AAS o dabigatrán hasta cumplir 35 días posoperatorios, seguimiento mínimo de 90 días. 
Los criterios de exclusión fueron: artroplastias como tratamiento de fractura y artroplastia de revisión, cuadros clínicos que contraindican el uso de AAS o dabigatrán, anticoagulación previa, antecedentes de neoplasia o de TVP/TEP, índice de masa corporal $>35$, artroplastias bilaterales y simultáneas, evento trombótico durante la estancia hospitalaria, ya que, en ese lapso, los pacientes reciben profilaxis con enoxaparina subcutánea 0,4 cc/día; fracturas intraoperatorias asociadas, estancia hospitalaria $>6$ días, nueva cirugía no relacionada con la intervención en estudio que haya requerido reinternación durante el seguimiento y pérdida del seguimiento.

Los pacientes se internaron el mismo día de la cirugía programada y fueron evaluados diariamente junto con los profesionales del Servicio de Clínica Médica.

Las medidas profilácticas antitrombóticas adoptadas fueron:

- Enoxaparina subcutánea 0,4 cc/día durante la internación, rotando a la vía oral el día del alta hospitalaria.

- Vendaje compresivo en ambos miembros inferiores colocado en el posoperatorio inmediato. Alta hospitalaria con medias de compresión de uso diurno.

- Movilización precoz en el posoperatorio inmediato y marcha a las 12-24 h con andador o bastones canadienses según la tolerancia.

Tras el alta hospitalaria, los pacientes continuaron la profilaxis antitrombótica por vía oral, en dos esquemas diferentes. En el grupo I, se utilizó AAS $325 \mathrm{mg}$ en una única dosis diaria. En el grupo II, se indicó dabigatrán en dosis ajustadas según la edad (<75 años, $220 \mathrm{mg} /$ día y > 75 años, $150 \mathrm{mg}$ /día) y la depuración de creatinina. El tratamiento profiláctico antitrombótico continuó de esta manera en el domicilio hasta el día 35 posterior a la cirugía.

Se realizaron controles seriados clínicos y radiográficos a los 10 días, 3, 6 y 12 semanas de la cirugía con la finalidad de evaluar la evolución y detectar complicaciones.

Se registraron las complicaciones que ocurrieron durante los primeros 90 días posteriores a la operación, inclusive ETE sintomático (TVP y TEP), complicaciones hemorrágicas, de la herida quirúrgica, infección aguda de la prótesis y muerte.

Ante la sospecha clínica de TVP, se confirmó o descartó mediante eco-Doppler venoso en el momento de la consulta. Si se sospechaba TEP, se realizó una angiotomografía computarizada pulmonar. En ambas situaciones, si el resultado era positivo, se iniciaba el tratamiento anticoagulante correspondiente. Si se descartaban TVP o TEP, se continuaba con el tratamiento profiláctico en curso.

La infección aguda de la prótesis se confirmó teniendo en cuenta parámetros clínicos y de laboratorio, según las recomendaciones de la Musculoskeletal Infection Society.

El costo del tratamiento profiláctico antitrombótico para ambos grupos se calculó en dólares estadounidenses (USD), según la cotización oficial del Banco de la Nación Argentina, el 15 de abril de 2020, a USD 66,75 de la terapia antitrombótica hasta completar los 35 días posoperatorios.

Este estudio fue aprobado por el Comité de Bioética de nuestro Centro.

\section{Análisis estadístico}

Las variables continuas se describieron como media y desviación estándar, y las variables cualitativas, como porcentaje e intervalo de confianza del $95 \%$. Se compararon los dos grupos de análisis mediante una prueba t para las variables cuantitativas y la prueba de Fisher para las variables cualitativas. Se consideró estadísticamente significativa una diferencia $<0,05$. Se utilizó el programa Graph Pad Prism 8.0.

\section{RESULTADOS}

Entre el 1 de mayo de 2019 y el 20 de abril de 2020, se realizaron 353 artroplastias primarias de cadera y rodilla. Se excluyó a 126 pacientes, porque no cumplían los criterios de inclusión, alguno de ellos no cumplían con más de un criterio (Figura 1).

La serie quedó conformada por 224 pacientes, con una media de la edad de 68.5 años (rango 38-95). Ciento veinticinco $(55,8 \%)$ eran mujeres y $99(44,2 \%)$, hombres, con un índice de masa corporal de 28,1 (rango 17-35). Los procedimientos fueron $115(51,3 \%)$ RTC y 109 RTR $(48,7 \%)$ y el tiempo promedio de internación fue de 3.2 días (rango 2-6).

Tras el alta, 80 pacientes continuaron la tromboprofilaxis por vía oral con AAS $(35,7 \%)$ y 144, con dabigatrán $(64,3 \%)$ (Tabla 1). No se observaron diferencias estadísticamente significativas en las características demográficas de ambos grupos (Tabla 2). 


\section{Artroplastias de cadera y de rodilla \\ $\mathrm{n}=353$}

\section{Exclusión preoperatoria}

- Trastornos hemáticos $(n=17)$

- Antecedentes oncológicos $(n=24)$

- Cuadros clínicos ( $n=19)$

- Tratamiento de fractura $(n=50)$

\section{Exclusión posoperatoria}

- Reemplazo articular bilateral/simultáneo $(\mathrm{n}=9)$

- Internación prolongada $(n=4)$

- Pérdida de seguimiento $(n=3)$

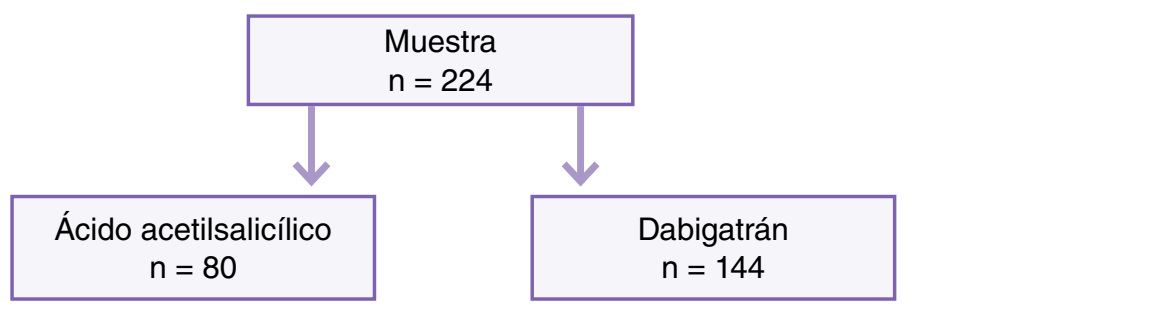

Figura 1. Diagrama de exclusión de los pacientes.

Tabla 1. Características demográficas de la serie

\section{Muestra: 224}

Edad

Índice de masa corporal

Seguimiento

Días de internación

Sexo

Procedimiento quirúrgico

Reemplazo total de cadera

Reemplazo total de rodilla

Profilaxis antitrombótica

Ácido acetilsalicílico

Dabigatrán
68.5 (rango 38-95)

28.1 (rango 17-35)

3 meses

3.2 (rango 2-6)

Masculino: $99(44,2 \%)$

Femenino: 125 (55,8\%)

$115(51,3 \%)$

$109(48,7 \%)$

$80(35,7 \%)$

$114(64,3 \%)$

Tabla 2. Características demográficas según la profilaxis antitrombótica

\begin{tabular}{|l|c|c|c|}
\hline Muestra: 224 & Ácido acetilsalicílico $(\mathbf{n}=\mathbf{8 0})$ & Dabigatrán $(\mathbf{n}=\mathbf{1 4 4})$ & $\mathbf{p}$ \\
\hline Edad (años) & $69.7 \pm 8.8$ & $67.8 \pm 10.4$ & 0,2 \\
\hline Sexo & $\begin{array}{c}\text { Masculino }=33 \\
\text { Femenino }=47\end{array}$ & $\begin{array}{c}\text { Masculino }=66 \\
\text { Femenino }=78\end{array}$ & 0,5 \\
\hline Índice de masa corporal & $28 \pm 3,9$ & $28 \pm 3,8$ & 0,9 \\
\hline Días de internación & $3.2 \pm 0.5$ & $3.3 \pm 0.6$ & 0,7 \\
\hline Reemplazo total de rodilla/cadera & $44(55 \%) / 36(45 \%)$ & $66(45 \%) / 78(55 \%)$ & 0,6 \\
\hline
\end{tabular}


En general, la tasa de complicaciones extrahospitalarias fue del $8 \%(\mathrm{n}=18)$; la incidencia total de ETE sintomático fue del 1,3\%. Tres pacientes sufrieron TVP confirmada por eco-Doppler venoso, todos hombres y $>70$ años.

Según el tipo de procedimiento, la incidencia de ETE fue del 1,7\% en el RTC ( 2 casos) y del $0,9 \%$ en el RTR (1 caso), sin significancia estadística (p 0,8).

$\mathrm{Si}$ analizamos los grupos por separado, de acuerdo con el tratamiento tromboprofiláctico, la incidencia de TVP fue del 1,3\% para el grupo I ( 1 caso) y del $1,4 \%$ para el grupo II ( 2 casos).

Los casos de TVP en pacientes tratados con dabigatrán ocurrieron a los 16 y 30 días de la cirugía, es decir, mientras el paciente aún recibía profilaxis antitrombótica. En el caso tratado con AAS, el ETE tuvo lugar a los 60 días de la operación, ya finalizados los 35 días de tromboprofilaxis. No se observaron diferencias estadísticamente significativas en la incidencia de ETE entre ambos grupos $(\mathrm{p}=0,9)$.

Otros siete pacientes se presentaron con sintomatología compatible con TVP que luego fue descartada por ecoDoppler venoso, de manera que continuaron el esquema de tromboprofilaxis previo (4 con dabigatrán y 3 con AAS).

Con respecto a las complicaciones infecciosas y la tasa de mortalidad, si comparamos ambos grupos, la incidencia de infección superficial de la herida quirúrgica fue del 1,3\% en el grupo de AAS y del 3,5\% en el de dabigatrán $(\mathrm{p}=0,3)$ que fue tratada con antibióticos orales, a pesar de no ser estadísticamente significativo, fue porcentualmente mayor en el grupo II.

Se diagnosticaron dos infecciones agudas de la prótesis $(0,9 \%)$ tratadas con limpieza quirúrgica y buena evolución, en el grupo II, y ninguna en el grupo I ( $\mathrm{p}=0,9)$, lo que no fue estadísticamente significativo.

En el grupo I, se registró una muerte súbita $(0,4 \%)$ de causa no determinada a los 45 días de la cirugía, una vez finalizada la tromboprofilaxis con AAS $(\mathrm{p}=0,7)$.

No se observaron complicaciones hemorrágicas mayores. No hubo diferencias estadísticamente significativas en la incidencia del resto de las complicaciones entre ambos grupos (Tabla 3 y Figura 2).

Tabla 3. Complicaciones posoperatorias según la profilaxis antitrombótica

\begin{tabular}{|l|c|c|c|}
\hline Muestra: $\mathbf{2 2 4}$ & Ácido acetilsalicílico $(\mathbf{n}=\mathbf{8 0})$ & Dabigatrán $(\mathbf{n}=\mathbf{1 4 4})$ & $\mathbf{p}$ \\
\hline Evento tromboembólico & $1(1,3 \%)$ & $2(1,4 \%)$ & 0,9 \\
\hline Infección superficial & $1(1,3 \%)$ & $5(3,5 \%)$ & 0,3 \\
\hline Infección de la prótesis & 0 & $2(1,4 \%)$ & 0,7 \\
\hline Dehiscencia de la herida & $1(1,3 \%)$ & $1(0,7 \%)$ & 0,7 \\
\hline Rigidez articular & 0 & $2(1,4 \%)$ & 0,9 \\
\hline Lesión del aparato extensor & $1(1,3 \%)$ & 0 & 0,7 \\
\hline Muerte & $1(1,25 \%)$ & 0 & 0,3 \\
\hline Eventos totales & $6(7,5 \%)$ & $12(8,3 \%)$ & 0,9 \\
\hline
\end{tabular}

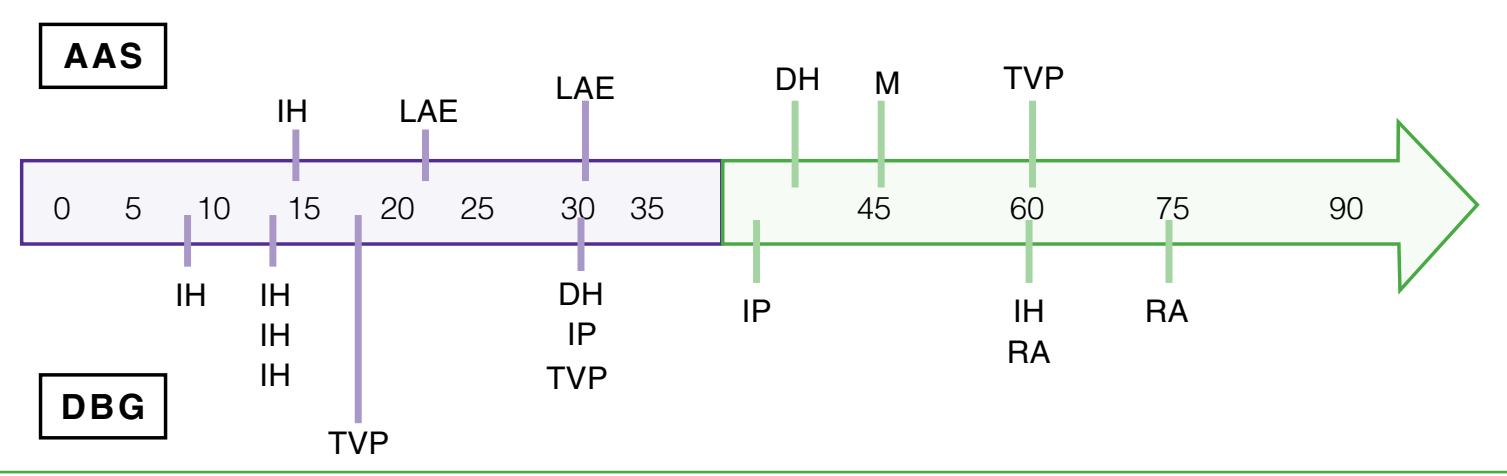

Figura 2. Distribución cronológica de las complicaciones (en días). AAS = ácido acetilsalicílico, DBG = dabigatrán, $\mathrm{IH}=$ infección de herida, LAE = lesión del aparato extensor, TVP = trombosis venosa profunda, DH = dehiscencia de la herida, $\mathrm{IP}=$ infección de la prótesis, $\mathrm{RA}=$ rigidez articular, $\mathrm{M}=$ muerte. 
En lo referente a la accesibilidad del tratamiento, el costo de la tromboprofilaxis con AAS (30 comprimidos con cobertura entérica $325 \mathrm{mg}$ ) durante 31 días fue de USD 3,6 mientras que, con dabigatrán, fue de USD 130 o 175, en función de su presentación, de 75 y 110 mg, respectivamente (cotización al 15/4/2020 BNA a 66.75 pesos argentinos).

\section{DISCUSIÓN}

El AAS fue incorporado como recomendación en la profilaxis antitrombótica para el período posoperatorio del RTC y RTR por la guía de la American Academy of Orthopaedic Surgeons ${ }^{9}$ y posteriormente, por la guía del American College of Chest Physicians para su uso como profilaxis de ETE. ${ }^{10}$ Desde entonces, el porcentaje de cirujanos que decide emplearla como único fármaco para la tromboprofilaxis aumentó de forma significativa. ${ }^{4}$

En 2018, la guía europea del National Institute of Health and Care Excellence (NICE) incorpora el AAS como recomendación en la profilaxis antitrombótica tras una artroplastia total de rodilla y como profilaxis extendida en la artroplastia total de cadera. ${ }^{14,15}$ Sin embargo, no hay un consenso actual en cuanto a la dosis idónea y su tiempo de uso. ${ }^{1,7,10,16}$

En nuestro estudio, decidimos administrar $325 \mathrm{mg}$ como dosis diaria de AAS, porque autores, como Parvizi y cols. ${ }^{7}$ no hallaron diferencias significativas en la prevención del TEP con la administración de AAS a bajas dosis ( $81 \mathrm{mg}, 2$ veces/día) o altas dosis ( $325 \mathrm{mg}$, 2 veces/día) durante cuatro semanas, ni en la incidencia de eventos gastrointestinales, infección de la prótesis o la muerte, así como tampoco Feldstein y cols. ${ }^{16}$ entre otros. ${ }^{3}$ Preferimos esa dosis para disminuir el riesgo de hematomas y sangrado por la herida. Esta decisión se condice con nuestros resultados, ya que no hemos registrado complicaciones hemorrágicas ni hematomas con AAS en dosis de $325 \mathrm{mg}$ por día.

En 2000, se publican los resultados de un estudio aleatorizado doble ciego, el ensayo Pulmonary Embolism Prevention (PEP), ${ }^{11}$ en el cual se evalúa la incidencia de ETE en más de 17.000 pacientes tratados con AAS o placebo por fractura de cadera o artroplastia programada de rodilla y de cadera, y se llegó a la conclusión de que el AAS reduce el riesgo de TEP y TVP en, al menos, un 30\% en los primeros 35 días después de la intervención.

En nuestro estudio, la incidencia de ETE fue similar entre los pacientes que recibieron AAS o dabigatrán. La incidencia total de ETE sintomático en general, durante los primeros 90 días del posoperatorio fue del 1,3\%, y siempre se trató de TVP. La incidencia de esta complicación en el grupo de pacientes tratados con AAS y en los que recibieron dabigatrán no fue estadísticamente significativa. Esto mismo tampoco se verificó entre ambos procedimientos, RTC y RTR, tal como se informa en distintas publicaciones que evalúan ambos procedimientos por igual, ya que se utilizan los mismos agentes como tromboprofilaxis para ambas. ${ }^{3-5,8,16}$

Cabe destacar que, en nuestro estudio, las TVP en el grupo de dabigatrán ocurrieron a los 16 y 30 días de la cirugía, es decir, durante el período de tromboprofilaxis, mientras que, en el grupo con AAS, se produjeron a los 60 días, 25 días después de finalizar la farmacoprofilaxis.

En un estudio multicéntrico sobre la incidencia de ETE, Bozic y cols. ${ }^{17}$ evaluaron a 93.840 pacientes sometidos a RTR que recibieron AAS, warfarina o heparina de bajo peso molecular, y las tasas de ETE fueron del $2,3 \%$; $4 \%$ y $3,1 \%$, respectivamente. Por otro lado, Ogonda y cols., ${ }^{8}$ en un estudio prospectivo de 11.459 pacientes sometidos a RTR, RTC o reemplazo unicompartimental de rodilla, comprobaron la seguridad del AAS como agente tromboprofiláctico, ya que no aumentó el riesgo de muerte, TEP o TVP en pacientes de bajo riesgo; la incidencia total fue del $0,3 \%$ de TVP y $0,6 \%$ de TEP en RTC, del 1,5\% en RTR y del 1,2\% en el reemplazo unicompartimental de rodilla. En una revisión sistemática de 39 artículos, Vincent y cols. ${ }^{3}$ evaluaron los resultados en relación con el ETE de 69.551 pacientes sometidos a RTC y RTR que recibieron AAS como farmacoprofilaxis antitrombótica, y obtuvieron una tasa de TEP del 0,6\% y de TVP del 1,2\%, datos comparables a los obtenidos en nuestra serie.

McHale y cols. ${ }^{5}$ compararon retrospectivamente la incidencia de ETE en dos grupos de pacientes, uno tratado con dabigatrán $220 \mathrm{mg} /$ día, por 28 días para el RTC y 10 días para el RTR, frente a otro en el que se administró AAS $150 \mathrm{mg} /$ día, durante seis semanas para el RTC y el RTR. No comunicaron ETE en el grupo de pacientes con AAS, mientras que la incidencia de TVP fue del 2,2\% y 1,6\% para el RTC y el RTR, respectivamente, en el grupo con dabigatrán.

En cuanto a las complicaciones por infección, cinco de seis casos de infecciones superficiales de la herida y los dos casos de infección aguda de la prótesis se produjeron en el grupo con dabigatrán. Sin embargo, esta diferencia no fue estadísticamente significativa entre ambos grupos. Hay evidencia que asocia el drenaje persistente de la herida quirúrgica con un mayor tiempo de internación y riesgo de infección de la prótesis..$^{17,18,19}$ Parvizi y cols. ${ }^{18}$ 
hallaron una relación significativa entre el exceso de anticoagulantes y las complicaciones de la herida en pacientes que posteriormente tuvieron una infección de la prótesis. Por otro lado, en su estudio prospectivo, Aquilina y cols. ${ }^{19}$ comunicaron que el tiempo promedio de drenaje por la herida quirúrgica tras un RTC y un RTR fue de 6.4 días para el grupo con dabigatrán y de 3.2 días para el que recibió AAS, esto último no fue verificado en nuestro estudio. Otros estudios asocian a los agentes anticoagulantes habitualmente prescritos con tasas más altas de complicaciones hemorrágicas y débito persistente por la herida quirúrgica en relación con el AAS, ${ }^{20,21}$ tal como se mencionó antes.

En nuestra serie, se produjo una muerte súbita a las seis semanas del RTC (hombre de 72 años). El riesgo de muerte luego de este procedimiento varía según el tiempo transcurrido desde la cirugía, es más alto en los primeros 30 días y retorna al riesgo basal preoperatorio luego de los 90 días. ${ }^{22}$ Se dispone de fuerte evidencia que sugiere que la edad avanzada y el sexo masculino predisponen a sufrir una muerte prematura luego de un RTC. ${ }^{22,23}$ Asimismo, se acepta que las enfermedades cardiovasculares, como infarto agudo de miocardio o insuficiencia cardíaca, son responsables de más del $50 \%$ de las muertes, seguidas de la enfermedad cerebrovascular y el TEP. ${ }^{22,23}$ Bayley $^{2}$ y cols. ${ }^{24}$ refieren que el 11,7-17,1\% de los óbitos dentro de los 90 días posteriores a un RTC se deben a TEP. No obstante, la verdadera causa de muerte, en ocasiones, no puede ser identificada y numerosos estudios sugieren que, en estos casos, podría corresponder a TEP. ${ }^{1,7}$ En nuestro caso, no fue posible determinar el motivo de la muerte de este paciente.

Nuestros pacientes permanecieron internados una media de 3.2 días, lo que implica que la administración de AAS o dabigatrán se prolongó por una media de 31 días, hasta cumplir un total de 35 días de profilaxis. ${ }^{16}$ Es importante remarcar el enfoque multimodal de la profilaxis antitrombótica utilizado, teniendo en cuenta la administración de enoxaparina por vía subcutánea durante la internación, la farmacoprofilaxis oral al alta, los protocolos de movilización precoz y el uso de compresión mecánica en los miembros inferiores. ${ }^{1}$

En cuanto al costo de la profilaxis antitrombótica oral, hasta cumplir los 35 días desde la cirugía, fue de USD 3,6 con AAS y de USD 130 o 175 con dabigatrán, según la presentación (75 y $110 \mathrm{mg}$, respectivamente). La profilaxis del TEP y la TVP con AAS en pacientes después de un RTC y un RTR se puede presentar como una alternativa económica e igual de eficaz que el dabigatrán, fácil de conseguir, lo que permite lograr una mayor adhesión al tratamiento por parte de los pacientes. . $^{1,2,16,17,25}$

Las debilidades de este estudio son la reducida cantidad de pacientes que conforman la muestra y que no fue a doble ciego. Algunos pacientes tenían más de un criterio de exclusión. También cabe mencionar que se realizaron estudios de eco-Doppler solo a pacientes sintomáticos, sin considerar las TVP subclínicas. Además, los dos grupos fueron tratados por distintos cirujanos.

Sin embargo, sus fortalezas son que solo tres pacientes fueron excluidos por pérdida del seguimiento (Figura 1); además, todos fueron operados mediante el mismo abordaje, los mismos tipos de implantes, y el mismo protocolo anestésico y de rehabilitación; este estudio es inédito en nuestro país.

\section{CONCLUSIONES}

La administración de AAS como tromboprofilaxis tras un RTC o RTR a pacientes con bajo riesgo de ETE ha logrado resultados clínicos similares a los del dabigatrán. Se puede recomendar un protocolo multimodal basado en el uso de AAS. Este protocolo puede mejorar el cumplimiento de la terapia antitrombótica por parte de los pacientes, debido al bajo costo de la profilaxis con AAS al compararla con la de dabigatrán.

Queda como desafío la necesidad de realizar un estudio prospectivo, aleatorizado y doble ciego para reforzar estos resultados.

Conflicto de intereses: Los autores no declaran conflictos de intereses.

ORCID de T. Dainotto: https://orcid.org/0000-0002-6645-9928

ORCID de S. Gaggiotti: https://orcid.org/0000-0003-4077-2892

ORCID de H. del Sel: https://orcid.org/0000-0002-3655-1408 


\section{BIBLIOGRAFÍA}

1. Azboy I, Barrack R, Thomas AM, Haddad FS, Parvizi J. Aspirin and the prevention of venous thromboembolism following total joint arthroplasty: Commonly asked questions. Bone Joint J 2017;99B(11):1420-30. https://doi.org/10.1302/0301-620X.99B11.BJJ-2017-0337.R2

2. Mostafavi Tabatabaee R, Rasouli MR, Maltenfort MG, Parvizi J. Cost-effective prophylaxis against venous thromboembolism after total joint arthroplasty: warfarin versus aspirin. J Arthroplasty 2015;30(2):159-64. https://doi.org/10.1016/j.arth.2014.08.018

3. An VVG, Phan K, Levy YD, Bruce WJM. Aspirin as thromboprophylaxis in hip and knee arthroplasty: a systematic review and meta-analysis. J Arthroplasty 2016;31(11):2608-16. https://doi.org/10.1016/j.arth.2016.04.004

4. Shah SS, Satin AM, Mullen JR, Merwin S, Goldin M, Sgaglione NA. Impact of recent guideline changes on aspirin prescribing after knee arthroplasty. J Orthop Surg Res 2016;11(1):123. https://doi.org/10.1186/s13018-016-0456-0

5. McHale S, Williams M, O'Mahony C, Hockings M. Should we use dabigatran or aspirin thromboprophylaxis in total hip and knee arthroplasty? A natural experiment. J Orthop 2019;16(6):563-8. https://doi.org/10.1016/j.jor.2019.05.008

6. Lee YK, Chung CY, Koo KH, Lee KM, Ji HM, Park MS. Conflict of interest in the assessment of thromboprophylaxis after total joint arthroplasty: A systematic review. J Bone Joint Surg Am 2012;94(1):27-33. https://doi.org/10.2106/JBJS.J.01033

7. Parvizi J, Huang R, Restrepo C, Chen AF, Austin MS, Hozack WJ, et al. Low-dose aspirin is effective chemoprophylaxis against clinically important venous thromboembolism following total joint arthroplasty a preliminary analysis. J Bone Joint Surg Am 2017;99(2):91-8. https://doi.org/10.2106/JBJS.16.00147

8. Ogonda L, Hill J, Doran E, Dennison J, Stevenson M, Beverland D. Aspirin for thromboprophylaxis after primary lower limb arthroplasty. Bone Joint J 2016;98B(3):341-8. https://doi.org/10.1302/0301-620X.98B3.36511

9. Mont MA, Jacobs JJ. AAOS clinical practice guideline: preventing venous thromboembolic disease in patients undergoing elective hip and knee arthroplasty. J Am Acad Orthop Surg 2011;19(12):768-76. https://doi.org/10.5435/00124635-201112000-00008

10. Falck-Ytter Y, Francis CW, Johanson NA, Curley C, Dahl OE, Schulman S, et al. Prevention of VTE in orthopedic surgery patients. Antithrombotic therapy and prevention of thrombosis, 9th ed. American College of Chest Physicians evidence-based clinical practice guidelines. Chest 2012;141(2 Suppl):e278S-e325S. https://doi.org/10.1378/chest.11-2404

11. Rodgers A, MacMahon S, Collins R, Prentice C. Prevention of pulmonary embolism and deep vein thrombosis with low dose aspirin: Pulmonary Embolism Prevention (PEP) trial. Lancet 2000;355(9212):1295-302. PMID: 10776741

12. Lussana F, Squizzato A, Permunian Tamborini E, Cattaneo M. A systematic review on the effect of aspirin in the prevention of post-operative arterial thrombosis in patients undergoing total hip and total knee arthroplasty. Thromb Res 2014;99-B(11):1420-30. PMID: 29092979

13. Eriksson BI, Dahl OE, Rosencher N, Kurth AA, van Dijk CN, Frostick SP, et al. RE NOVATE (Dabigatran v warfarin hip). Lancet 2007;370(9591):949-56. https://doi.org/10.1016/S0140-6736(07)61445-7

14. Jenny JY, Pabinger I, Samama CM. European guidelines on perioperative venous thromboembolism prophylaxis. Eur J Anaesthesiol 2018;35(2):123-9. https://doi.org/ 10.1097/EJA.0000000000000728

15. NICE Guidance. Venous thromboembolism in over 16s: reducing the risk of hospital-acquired deep vein thrombosis or pulmonary embolism. Natl Inst Heal Care Excell 2018. Disponible en: https://www.nice.org.uk/guidance/ng89

16. Feldstein MJ, Low SL, Chen AF, Woodward LA, Hozack WJ. A Comparison of two dosing regimens of ASA following total hip and knee arthroplasties. J Arthroplasty 2017;32(9):S157-61. https://doi.org/10.1016/j.arth.2017.01.009

17. Bozic KJ, Vail TP, Pekow PS, Maselli JH, Lindenauer PK, Auerbach AD. Does aspirin have a role in venous thromboembolism prophylaxis in total knee arthroplasty patients? J Arthroplasty 2010;25(7):1053-60. https://doi.org/10.1016/j.arth.2009.06.021

18. Parvizi J, Ghanem E, Joshi A, Sharkey PF, Hozack WJ, Rothman RH. Does “excessive” anticoagulation predispose to periprosthetic infection? J Arthroplasty 2007;22(6 Suppl 2):24-8. https://doi.org/ 10.1016/j.arth.2007.03.007

19. Aquilina AL, Brunton LR, Whitehouse MR, Sullivan N, Blom AW. Direct thrombin inhibitor (DTI) vs. aspirin in primary total hip and knee replacement using wound ooze as the primary outcome measure. A prospective cohort study. Hip Int 2012;22(1):22-7. PMID: 22362503 
20. Lindquist DE, Stewart DW, Brewster A, Waldroup C, Odle BL, Burchette JE, et al. Comparison of postoperative bleeding in total hip and knee arthroplasty patients receiving rivaroxaban, enoxaparin, or aspirin for thromboprophylaxis. Clin Appl Thromb 2018;24(8):1315-21. https://doi.org/10.1177/1076029618772337

21. Wilson DGG, Poole WEC, Chauhan SK, Rogers BA. Systematic review of aspirin for thromboprophylaxis in modern elective total hip and knee arthroplasty. Bone Joint J 2016;98-B(8):1056-61. https://doi.org/10.1302/0301-620X.98B8.36957

22. Hunt LP, Ben-Shlomo Y, Clark EM, Dieppe P, Judge A, MacGregor AJ, et al. 90-day mortality after 409096 total hip replacements for osteoarthritis, from the National Joint Registry for England and Wales: A retrospective analysis. Lancet 2013;382(9898):1097-104. https://doi.org/10.1016/S0140-6736(13)61749-3

23. Singh JA, Lewallen DG. Ninety-day mortality in patients undergoing elective total hip or total knee arthroplasty. $J$ Arthroplasty 2012;27(8):1417-22. https://doi.org/ 10.1016/j.arth.2012.03.008

24. Quah C, Bayley E, Bhamber N, Howard P. Fatal pulmonary embolism following elective total knee replacement using aspirin in multi-modal prophylaxis - A 12 year study. Knee 2017;24(5):1187-90. https://doi.org/10.1016/j.knee.2017.05.021

25. Gutowski CJ, Zmistowski BM, Lonner JH, Purtill JJ, Parvizi J. Direct costs of aspirin versus warfarin for venous thromboembolism prophylaxis after total knee or hip arthroplasty. J Arthroplasty 2015;30(9 Suppl):36-8. https://doi.org/10.1016/j.arth.2015.04.048 